\title{
Adapting the Outphasing Technique for VLC Based on Summing the Light
}

\author{
Daniel G. Aller, Diego G. Lamar, Juan Rodríguez, Pablo F. Miaja y Javier Sebastián. \\ \{garciaadaniel, gonzalezdiego, rodriguezmjuan, fernandezmiapablo, sebas\}@ uniovi.es \\ Electrical, Electronic, Computers and Systems Engineering Department, \\ University of Oviedo, Gijón 33204, Spain.
}

\begin{abstract}
:
This work proposes a high efficiency LED driver for Visible Light Communication (VLC) working as a transmitter based on the outphasing technique. Outphasing lays on the idea of reproducing a non-constant amplitude sine signal by adding two phase modulated sine signals with constant amplitude, which leads to an increase of the overall efficiency of the amplification stage. The proposed transmitter is made up of two switching-mode power amplifiers and a DC/DC converter. Each amplifier reproduces one of the two sine waves required to perform the communication signal using the outphasing technique and the DC/DC converter biases the LED strings. The proposal takes advantage of the light and instead of adding the signals electrically, the signals are adding in their light form, which leads to a reduction on the complexity of the design due to the electrical independency between the two amplifiers. As experimental results, a transmitter is made of two Class E amplifiers reproducing a 16-QAM modulation with a $5 \mathrm{MHz}$ carrier, achieving an efficiency of $78 \%$ in the signal generation (higher than the Class A and B maximum efficiency for a 16-QAM modulation) and a $92 \%$ in the overall efficiency when the communication and lighting tasks are taking into account (on the same page as the DC-DC converter alternatives).
\end{abstract}

\section{INTRODUCTION}

Nowadays, almost every wireless communication is based on the use of the Radio Frequency (RF) spectrum, which leads to a high congestion and a strictly regulation [1]. Due to these problems, some new techniques have been developed in the last few years in order to avoid the problem, and one of this alternatives is Visible Light Communication (VLC) [2]-[4] that uses the wide and unregulated visible light spectrum (from 430 to $750 \mathrm{THz}$ ). VLC goes hand in hand with the widespread use of the LED technology for Solid-State Lighting (SSL) applications by taking advantage of the fast light modulation capability of the LED.

The main idea behind VLC is the use of the SSL infrastructure to perform the lighting and the communication task at the same time. Figure 1 shows the current vs voltage and

This work was supported in part by the European Regional Development Fund grants, in part by the Spanish Government under the project MINECO17-DPI2016-75760-R, and in part by the Principality of Asturias under the project IDI/2018/000179 and under the scholarship BP17-91.

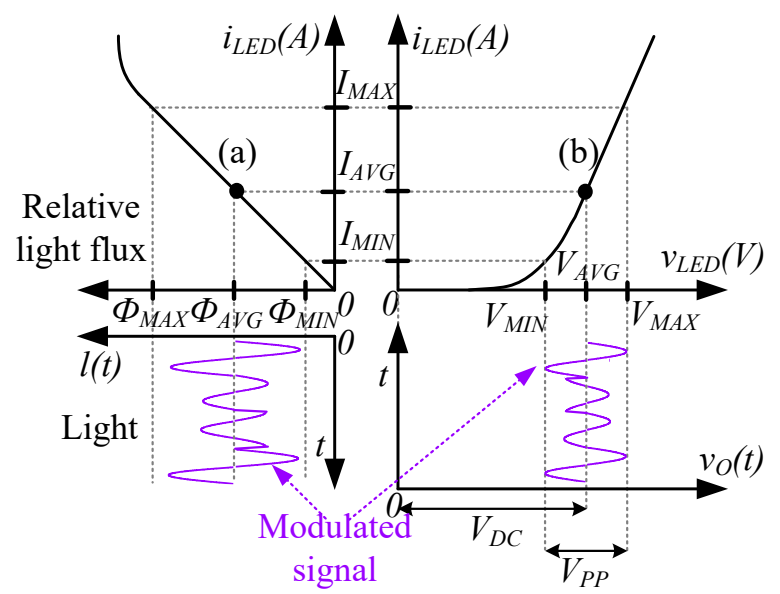

Fig. 1: A LED working as VLC transmitter in its current/voltage and light/current relation.

the light vs current relation on the LED. In order to fulfill the illumination task, an average voltage $V_{A V G}$ is applied across the LED in order to bias it, which leads to an average current $I_{A V G}$ flowing through the LED, emitting an average light intensity $\Phi_{A V G}$. The communication task is performed by a variation around the average value. As an example, a communication signal is applied as a voltage and it changes its amplitude (varying between $V_{M I N}$ and $V_{M A X}$ ) and its phase. That signal leads to a proportional variation of the current through the LED (between $I_{M I N}$ and $I_{M A X}$ ) and on the light emitted by the LED (between $\Phi_{M I N}$ and $\Phi_{M A X}$ ). It is important to emphasize that two different efficiencies are taken into account since each task can be performed by different circuitry: the signal generation efficiency and the overall efficiency. The signal generation efficiency only takes into account the efficiency of the communication circuitry, and the overall takes into account the efficiency of the communication and biasing circuits altogether.

Since one of the main advantages of SSL is its high efficiency, adding the communication capability must not deteriorate excessively the overall efficiency of the system. In the last few years, some VLC transmitter topologies have been proposed where a linear power amplifier performs the communication task (i.e. Class A, B or AB) [5]-[7], which leads to a low signal efficiency of the power amplifiers, 
which compromises the efficiency of the whole system. The theoretical maximum is $50 \%$ for Class A and $78.5 \%$ for Class $\mathrm{B}$, but for a non-constant amplitude modulation the efficiency drops significantly (i.e. for a 16-QAM digital modulation, the maximum efficiency is $25 \%$ for Class A and $55 \%$ for Class B).

On the other hand, the use of DC-DC converters as a VLC transmitters has been proposed to increase the power efficiency of the system [8]-[13]. The DC-DC converter generates the bias and the communication signal at the same time, reaching an overall power efficiency higher than $90 \%$ (communication and biasing task), but the disadvantages are the limitation on the maximum communication bandwidth, the high complexity of the DC-DC topologies and their complex control techniques.

Due to the disadvantages of both proposals, an adaptation of the well-known outphasing technique for VLC is proposed in this paper. The outphasing technique was first propossed in the 30's [14], [15] as a method to increase the efficiency of the RF amplification stage when the amplitude of the RF sine signal is not constant. Outphasing is based on the idea of splitting a non-constant amplitude sine signal into two phase modulated sine signals with constant amplitude, and making the amplification task over the later sine signals. Since linear amplifiers (i.e. Class A, B or AB) and resonant switchingmode amplifiers (i.e. Class E) can only achieve their maximum efficiency when the amplitude of the signal is constant, the efficiency of the amplification stage is improved.

In this paper, a VLC outphasing transmitter based on two Class E amplifiers is presented. Each Class E amplifier reproduces one of the two constant amplitude sine signals required for the outphasing. This proposal lays on the same idea as the original outphasing technique but by taking advantage of using the light intensity as communication signal. The proposed light-outphasing technique adds the two sine signals in their light form instead of electrically. Using the light to sum the phases leads to a electrical isolation between each Class E amplifier, which strongly simplifies the design of the overall transmission system. As experimental results, a transmitter is built in order to transmit a 16-QAM digital modulation with a $5 \mathrm{MHz}$ carrier, achieving a bit rate up to $4 \mathrm{Mbps}$ and at a distance up to $1 \mathrm{~m}$. The prototype reaches an electrical efficiency of $78 \%$ in the signal generation (higher than the Class A and B maximum efficiency for a 16-QAM modulation) and a $92 \%$ in the overall efficiency when the communication and lighting tasks are taking into account (on the same page as the DC-DC converter alternatives).

The paper is organized as follows. Firstly, a briefly explanation about the outphasing technique is introduced in section II. Then its adaptation to VLC is explained in section III. Section IV contains the prototype design and the experimental results. Finally, section V gives the conclusions.

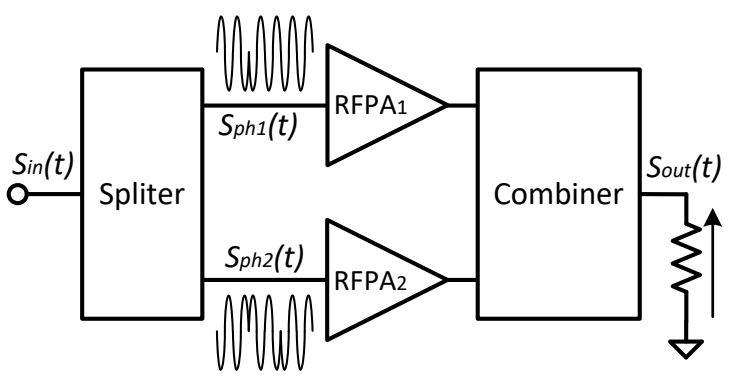

Fig. 2: Block diagram of a outpahsing RFPA made up of a spliter, two RFPAs and a combiner.

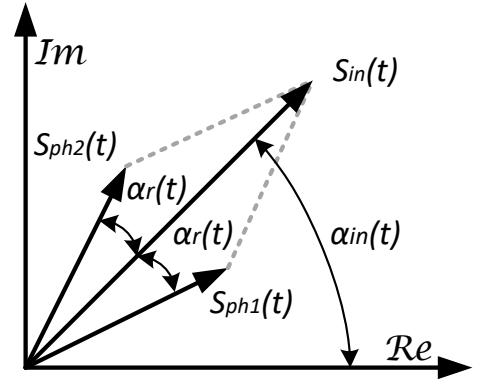

Fig. 3: Phasor diagram showing the amplitude and phase relation in the outphasing technique.

\section{OPERATING PRINCIPLE OF THE OUTPHASING TECHNIQUE}

Figure 2 shows the traditional outphasing implementation for RF power amplifiers. It is made up of a signal spliter, two Radio Frequency Power Amplifiers (RFPA) and a combiner.

\section{A. Outphasing mathematical analysis}

For the sake of simplicity, the communication signal $S_{i n}(t)$

$$
S_{i n}(t)=A_{i n}(t) \sin \left[2 \pi f_{s} t+\alpha_{i n}(t)\right]
$$

is a sine signal that varies its amplitude $A_{i n}(t)$ and phase $\alpha_{i n}(t)$. From $S_{i n}(t)$, the signal spliter generates the two constant amplitude phase modulated sine signals $S_{p h 1}(t)$ and $S_{p h 2}(t)$

$$
\begin{aligned}
& S_{p h 1}(t)=A_{p h} \sin \left[2 \pi f_{s} t+\alpha_{p h 1}(t)\right] \\
& S_{p h 2}(t)=A_{p h} \sin \left[2 \pi f_{s} t+\alpha_{p h 2}(t)\right]
\end{aligned}
$$

whose addition is $S_{i n}(t)$ and both have the same frequency $f_{s}$. Both signals also have the same constant amplitude $A_{p h}$, and phases $\alpha_{p h 1}(t)$ and $\alpha_{p h 2}(t)$ respectively that vary over time, as shown in Fig. 3. At this point, and because of the constant amplitude of $S_{p h 1}$ and $S_{p h 2}$, the RFPAs can be designed to operate in the point with the highest efficiency, and therefore, it increases the overall efficiency.

Assuming that both RFPAs have the same gain k, the signals after the amplification are $k S_{p h 1}$ and $k S_{p h 2}$. Since the output signal is defined as $S_{\text {out }}(t)=k S_{p h 1}+k S_{p h 2}$, the phase of $S_{\text {out }}(t)$ is the same as the input signal $S_{\text {in }}(t)$. The phases $\alpha_{p h 1}(t)$ and $\alpha_{p h 2}(t)$ can be written as a function of $\alpha_{i n}(t)$ and a relative phase $\alpha_{r}(t)$

$$
\begin{aligned}
& \alpha_{p h 1}(t)=\alpha_{i n}(t)-\alpha_{r}(t) \\
& \alpha_{p h 2}(t)=\alpha_{i n}(t)+\alpha_{r}(t)
\end{aligned}
$$


and the amplitude $A_{\text {out }}(t)$ can be obtained as a function of $\alpha_{p h}(t)$ by using trigonometry.

$$
A_{\text {out }}(t)=2 k A_{p h} \cos \left[\alpha_{r}(t)\right]
$$

The output signal is defined as follow:

$$
S_{\text {out }}(t)=A_{\text {out }}(t) \sin \left[2 p i f_{s} t+\alpha_{s}(t)\right]
$$

As a conclusion, the amplitude $A_{\text {out }}(t)$ depends on the relative phase $\alpha_{r}(t)$, the gain $\mathrm{k}$ of the RFPA and the amplitude $A_{p h}$, so the phase $\alpha_{s}(t)$ and the amplitude $A_{\text {out }}(t)$ of $S_{\text {out }}(t)$ can be controlled independently.

\section{LIGHT-OUTPHASING TECHNIQUE FOR VLC TRANSMITTERS}

The major difficulty in an outphasing RFPA is the design of the output combiner, which is in charge of connecting the two amplifiers together and summing the sine signals. The connection of the outputs of the RFPAs is not straightforward since the output impedance of each RFPA changes, leading to a undesirable influence between them [16]. That effect is specially critical when the RFPA is based on a resonant topology (i.e., Class E) in which the efficiency depends on the proper tunning of the resonant circuit. Therefore, in the case of outphasing technique, the resonant circuit is modified by the output impedance of the other amplifier. The analysis and design of the combiner has been carried out in [16]-[18], leading to complex mathematical analysis and circuitry.

The idea of light-outphasing is splitting the LED load between the two RFPAs and sum the two sine signals in their light form, as it can be seen in the block diagram in Fig. 4 and the output light signals in an example in Fig. 5. Both LED strings are biased using a external DC/DC converter which provides the $V_{A V G}$ value necessary to bias the LED strings. Because of the quantities added are two light intensities instead of two electrical signals, the need of using a combiner and connect electrically both amplifiers is avoided, leading to a huge simplification of the design and avoiding the influence between the output of both RFPAs (which increases the efficiency). The VLC transmitter is made up of two Class E RFPAs delivering the two signals $S_{p h 1}$ and $S_{p h 2}$ required for outphasing. Since the output of the Class E amplifiers are not connected together, the condition of Zero Voltage Switching (ZVS) of each amplifier does not depends on the other RFPA, simplifying the overall design.

\section{EXPERIMENTAL RESULTS}

In order to prove the concept of the light-outphasing technique, a prototype of an outphasing VLC transmitter made up of two Class E RFPA is shown in Fig. 6. Both Class E RFPA are designed identically and each amplifier is connected to an independent LED string made up of 8 XLamp $M X-3$ LEDs each. The strings are biased externally by a DC-DC converter that controls the average current through the LED string $\left(I_{A V G}=0.5 A\right)$. Controlling the average current across the strings ensures that the LED always works in its linear region regardless of the threshold voltage shift due to possible

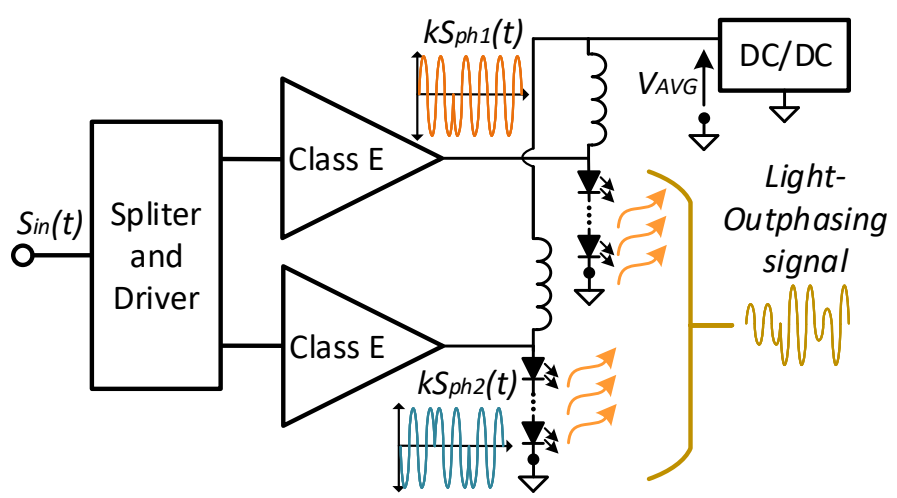

Fig. 4: Block diagram of a light-outphasing amplifier.

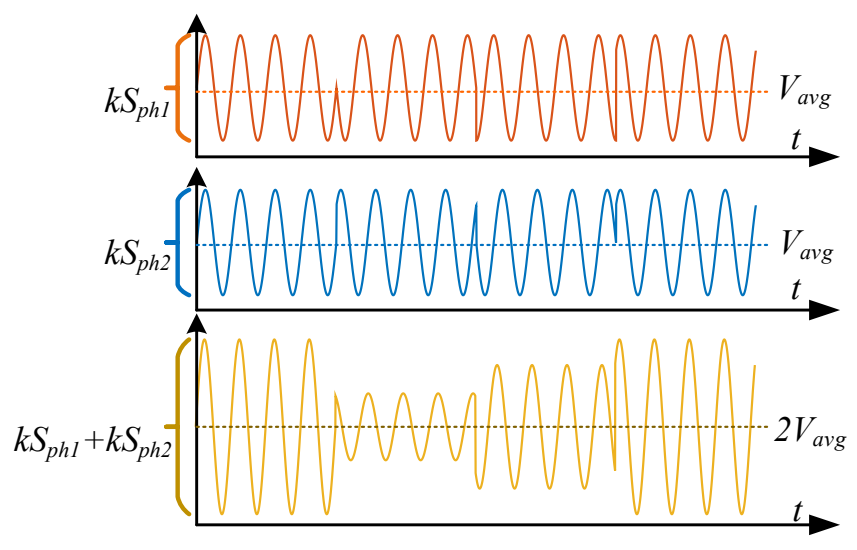

Fig. 5: Output signals in a the light-outphasing circuitry.

temperature changes on the LEDs. This allows the amplifier to apply the communication signal within the linear region of the LED, avoiding the undesirable distortion near the threshold voltage.

\section{A. Modulation Scheme}

In order to evaluate the communication task, a 16-QAM digital modulation with a carrier frequency of $5 \mathrm{MHz}$ is used. Each symbol of the modulation represents 4 bits and lasts 5 signal periods, providing a bit rate of $4 \mathrm{Mbps}$.

\section{B. Class E RFPA design}

Figure 7 shows the circuit of one of the two Class E RFPA that make up the outphasing transmitter, shown in Fig. 6. The amplifier is composed of a MOSFET, an output resonant circuit $\left(C_{1}, C\right.$ y $\left.L\right)$, a LED string and two biasing inductors $\left(L_{\text {bias }}, L_{c e}\right)$. The biasing inductors work as RF chokes that prevents the signal to pass through the biasing power supplies.

The amplifier is designed according to the modulation scheme presented before. The Class $\mathrm{E}$ is designed with a switching frequency $f_{s w}=5 \mathrm{MHz}$, and due to the high switching frequency required, a PD84010S-E RF MOSFET and a high speed EL7155 driver are used. As well as the switching frequency, the bandwidth necessary depends on the modulation. Even though the modulation is a 16-QAM, the signal delivered by each amplifier is only a phase modulation.

According to [19], a rough estimation of the bandwidth necessary for the modulation is $1.5 \mathrm{MHz}$. The bandwidth of 


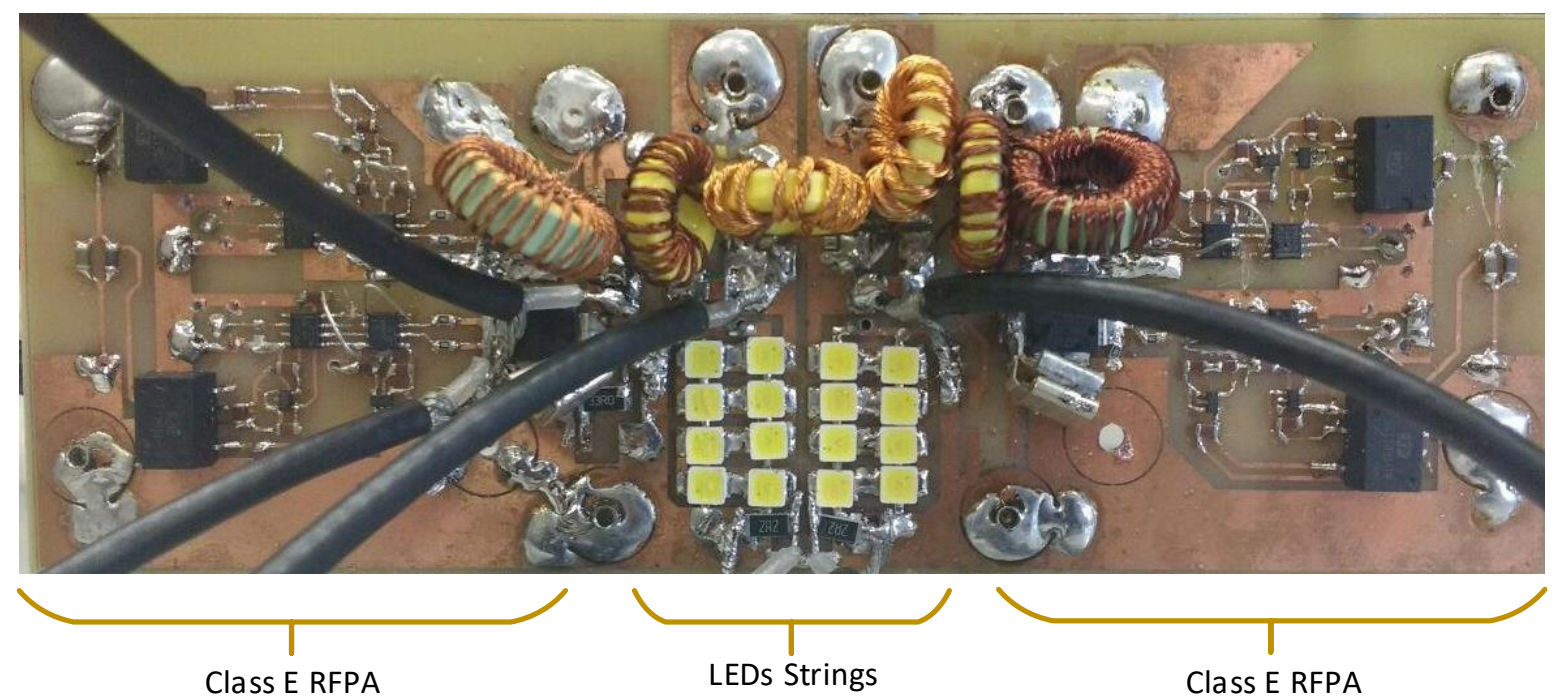

Fig. 6: Protype of the outphasing transmiter made up of two Class E amplifiers. Light-Outphasing.

the class $\mathrm{E}$ depends on the resonant output filter, as it can be seen in the Fig. 8, where the higher the bandwidth, the lower the quality factor Q of the filter. In order to properly reproduce the modulation, the bandwidth of the amplifier has to be higher than the bandwidth necessary for the modulation. Using the definition of $\mathrm{Q}$,

$$
Q=\frac{f_{s w}}{\Delta f_{s w}}=\frac{5 M H z}{1.5 M H z}=3.3
$$

the value be obtained from the switching frequency and the required bandwidth of the modulation. According to [20], [21], the resonant circuit values are shown in the table I.

Table I: COMPONENT VALUES OF THE RESONANT OUTPUT FILTER FOR THE CLASS E AMPLIFIER.

\begin{tabular}{ccc}
\hline$C_{1}$ & $C$ & $L$ \\
\hline $396 p F$ & $1.28 n F$ & $1.59 \mu H$ \\
\hline
\end{tabular}

\section{Experimental results}

Figure 9 depicts the light-outphasing process. Currents $I_{p h 1}(t)$ and $I_{p h 2}(t)$ are the currents through each LED string, which is proportional to the light that is emitted by each string. Since the amplitude of each sine current is kept constant, the

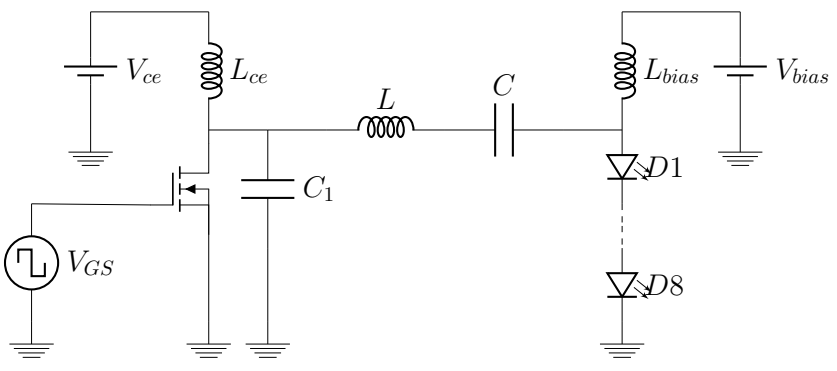

Fig. 7: Class E RFPA circuit.

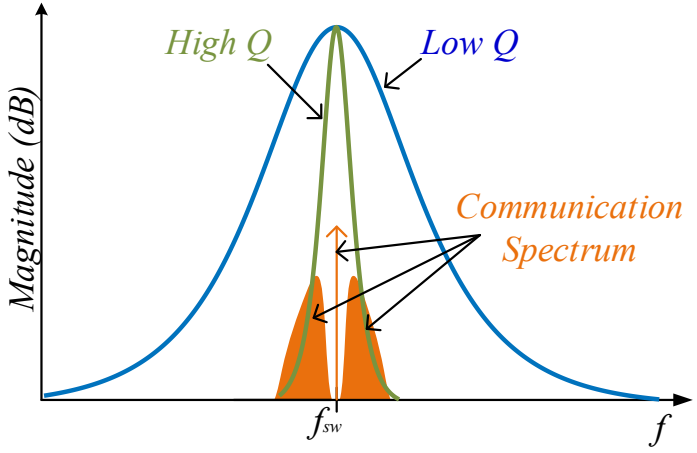

Fig. 8: Effect of the quality factor $Q$ of the filter communication signal in a Class E amplifier.

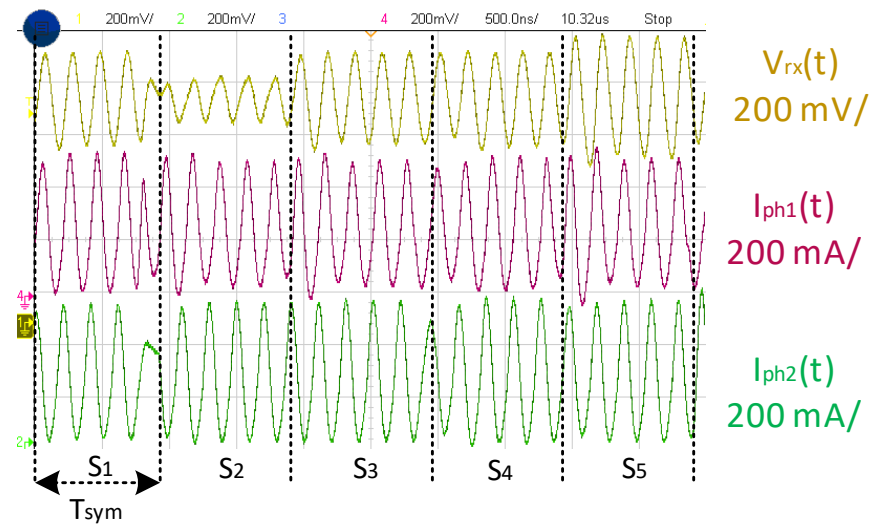

Fig. 9: Communication signals of the outphasing amplifier. $I_{p h 1}$ and $I_{p h 2}$ are the currents through each LED strings and $V_{r x}$ is the sum of the light.

amplitude of the light emitted by each string is constant as well, but due to the phase shift between them, when the light is added, the light received by a optical receiver $V_{r x}(t)$ reproduces the amplitude and phase changes of the modulation. The 
light is received by an optical receiver $P D A 10 A-E C$ placed in front of both strings.

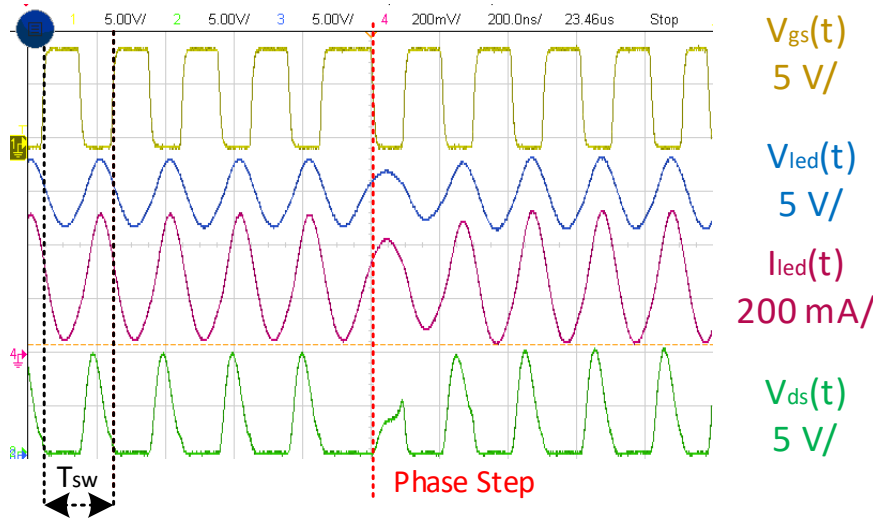

Fig. 10: Signals of one Class E RFPA when a phase change occurs. $V_{g s}$ is the gate signal and $V_{d s}$ is the drain to source voltaje. $V_{\text {led }}$ and $I_{l e d}$ are the voltaje across and the current through the LED string.

Figure 10 shows the main waveforms of the Class E RFPA when a change on the phase occurs. Before and after the phase change, the Class E RFPA works correctly, achieving ZVS and reproducing a sine signal. When a phase change occurs, during a switching period, the Class E losses ZVS, and takes two switching periods to achieve ZVS again. Due to this effect, there is a relation between the number of periods that each symbol lasts and the performance of the amplifier, the longer the symbol lasts, the higher the performance but the slower the bit rate. Because of this, a trade-off between performance and bit rate has to be established.

\section{CONCLUSIONS AND FUTURE WORK}

A VLC transmitter that is made of a DC/DC converter, two Class E amplifiers and two LED strings is presented in this work. The light-outphasing proposal consists in an adaptation of the outphasing technique for VLC. The two constant amplitude sine signals are added in their light form instead of adding the signals electrically, which leads to a major simplification of the circuitry and the design, as well as, an important improvement of the efficiency of the transmitter. Since the RFPAs are not connected together, there is no influence between them and the main disadvantage of the outphasing technique is avoided by using the light form of the signal. The proposed transmitter reproduces a 16-QAM digital modulation achieving a bit rate up to $4 \mathrm{Mbps}$ at a distance up to $1 \mathrm{~m}$. The prototype reaches an electrical efficiency of $78 \%$ in the signal generation (higher than the Class A and B maximum efficiency for a 16-QAM modulation) and a $92 \%$ in the overall efficiency when the communication and lighting tasks are taking into account (on the same page as the DC-DC converter alternatives).

\section{REFERENCES}

[1] Cisco Systems. (2017) Cisco visual networking index: Global mobile data traffic forecast update, 2016-2021.
[2] "IEEE standard for local and metropolitan area networks-part 15.7: Short-range wireless optical communication using visible light," IEEE Std 802.15.7-2011, pp. 1-309, Sept 2011.

[3] A. Jovicic, J. Li, and T. Richardson, "Visible light communication: opportunities, challenges and the path to market," IEEE Communications Magazine, vol. 51, no. 12, pp. 26-32, December 2013.

[4] H. Elgala, R. Mesleh, and H. Haas, "Indoor optical wireless communication: potential and state-of-the-art," IEEE Communications Magazine, vol. 49, no. 9, pp. 56-62, September 2011.

[5] H. Chun, S. Rajbhandari, G. Faulkner, D. Tsonev, E. Xie, J. J. D. McKendry, E. Gu, M. D. Dawson, D. C. O'Brien, and H. Haas, "LED based wavelength division multiplexed $10 \mathrm{~Gb} / \mathrm{s}$ visible light communications," Journal of Lightwave Technology, vol. 34, no. 13, pp. 3047-3052, July 2016.

[6] J. Vučić, C. Kottke, K. Habel, and K. D. Langer, "803 Mbit/s visible light WDM link based on DMT modulation of a single RGB LED luminary," in 2011 Optical Fiber Communication Conference and Exposition and the National Fiber Optic Engineers Conference, March 2011, pp. 1-3.

[7] F.-M. Wu, C.-T. Lin, C.-C. Wei, C.-W. Chen, Z.-Y. Chen, and H.T. Huang, "3.22-Gb/s WDM visible light communication of a single RGB LED employing carrier-less amplitude and phase modulation," in 2013 Optical Fiber Communication Conference and Exposition and the National Fiber Optic Engineers Conference (OFC/NFOEC), March 2013, pp. 1-3.

[8] J. Rodriguez, D. G. Lamar, P. F. Miaja, and J. Sebastian, "Reproducing single-carrier digital modulation schemes for VLC by controlling the first switching harmonic of the DC-DC power converter output voltage ripple," IEEE Transactions on Power Electronics, vol. 33, no. 9, pp. 7994-8010, Sept 2018.

[9] J. Rodriguez, D. G. Lamar, P. F. Miaja, D. G. Aller, and J. Sebastian, "Power efficient VLC transmitter based on pulse-width modulated DCDC converters and the split of the power," IEEE Transactions on Power Electronics, 2018.

[10] J. Sebastian, D. G. Lamar, D. G. Aller, J. Rodriguez, and P. F. Miaja, "On the role of power electronics in visible light communication," IEEE Journal of Emerging and Selected Topics in Power Electronics, pp. 1-1, 2018.

[11] J. Rodriguez, D. G. Lamar, D. G. Aller, P. F. Miaja, and J. Sebastian, "Efficient visible light communication transmitters based on switchingmode DC-DC converters," Sensors, vol. 18, no. 4, 2018.

[12] F. Loose, L. Teixeira, R. R. Duarte, M. A. Dalla Costa, and C. H. Barriquello, "On the use of the intrinsic ripple of a buck converter for visible light communication in led drivers," IEEE Journal of Emerging and Selected Topics in Power Electronics, vol. 6, no. 3, pp. 1235-1245, Sep. 2018.

[13] M. F. de Melo, J. S. Brand, R. R. Duarte, M. A. D. Costa, J. M. Alonso, and Y. Wang, "Analysis of low frequency ripple transmission in led drivers," in 2018 IEEE Industry Applications Society Annual Meeting (IAS), Sep. 2018, pp. 1-8.

[14] H. Chireix, "High power outphasing modulation," Proceedings of the Institute of Radio Engineers, vol. 23, no. 11, pp. 1370-1392, Nov 1935.

[15] L. F. Gaudernack, "A Phase-Opposition system of amplitude modulation," Proceedings of the Institute of Radio Engineers, vol. 26, no. 8, pp. 983-1008, Aug 1938.

[16] F. Raab, "Efficiency of outphasing RF Power-Amplifier systems," IEEE Transactions on Communications, vol. 33, no. 10, pp. 1094-1099, Oct 1985.

[17] M. Özen, M. van der Heijden, M. Acar, R. Jos, and C. Fager, "A generalized combiner synthesis technique for Class-E outphasing transmitters," IEEE Transactions on Circuits and Systems I: Regular Papers, vol. 64, no. 5, May 2017.

[18] R. A. Beltran and F. H. Raab, "Simplified analysis and design of outphasing transmitters using Class-E power amplifiers," in IEEE Topical Conference on Power Amplifiers for Wireless and Radio Applications (PAWR), Jan 2015.

[19] J. Proakis, Digital Communications, ser. Electrical engineering series. McGraw-Hill, 2008

[20] M. Kazimierczuk and K. Puczko, "Exact analysis of Class E tuned power amplifier at any Q and switch duty cycle," IEEE Transactions on Circuits and Systems, vol. 34, no. 2, pp. 149-159, February 1987.

[21] M. K. Kazimierczuk, "Class E tuned power amplifier with nonsinusoidal output voltage," IEEE Journal of Solid-State Circuits, vol. 21, no. 4, pp. 575-581, Aug 1986. 